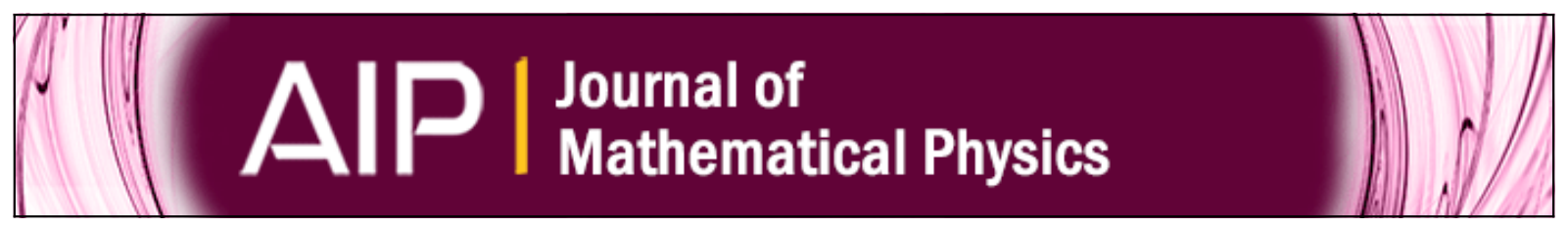

\title{
Parity-time symmetry broken by point-group symmetry
}

Francisco M. Fernández and Javier Garcia

Citation: Journal of Mathematical Physics 55, 042107 (2014); doi: 10.1063/1.4870642

View online: http://dx.doi.org/10.1063/1.4870642

View Table of Contents: http://scitation.aip.org/content/aip/journal/jmp/55/4?ver=pdfcov

Published by the AIP Publishing

\section{Articles you may be interested in}

Solving the Schrödinger eigenvalue problem by the imaginary time propagation technique using splitting methods with complex coefficients

J. Chem. Phys. 139, 124117 (2013); 10.1063/1.4821126

Quantum-dynamical consequences of the permutation symmetry of methyl groups

J. Chem. Phys. 132, 234301 (2010); 10.1063/1.3425880

Tracing molecular electronic excitation dynamics in real time and space

J. Chem. Phys. 132, 144302 (2010); 10.1063/1.3353161

A new variational method in the problem of the spectrum of elementary excitations in an edge-dislocation crystal Low Temp. Phys. 23, 976 (1997); 10.1063/1.593506

Displacement-operator squeezed states. I. Time-dependent systems having isomorphic symmetry algebras J. Math. Phys. 38, 84 (1997); 10.1063/1.531836

\section{AlP Re-register for Table of Content Alerts}




\title{
Parity-time symmetry broken by point-group symmetry
}

\author{
Francisco M. Fernández ${ }^{\mathrm{a})}$ and Javier Garcia \\ INIFTA (UNLP, CCT La Plata-CONICET), División Química Teórica, Blvd. 113 S/N, \\ Sucursal 4, Casilla de Correo 16, 1900 La Plata, Argentina
}

(Received 27 September 2013; accepted 23 March 2014; published online 21 April 2014)

\begin{abstract}
We discuss a parity-time (PT) symmetric Hamiltonian with complex eigenvalues. It is based on the dimensionless Schrödinger equation for a particle in a square box with the PT-symmetric potential $V(x, y)=$ iaxy. Perturbation theory clearly shows that some of the eigenvalues are complex for sufficiently small values of $|a|$. Pointgroup symmetry proves useful to guess if some of the eigenvalues may already be complex for all values of the coupling constant. We confirm those conclusions by means of an accurate numerical calculation based on the diagonalization method. On the other hand, the Schrödinger equation with the potential $V(x, y)=i a x y^{2}$ exhibits real eigenvalues for sufficiently small values of $|a|$. Point group symmetry suggests that PT-symmetry may be broken in the former case and unbroken in the latter one. (c) 2014 AIP Publishing LLC. [http://dx.doi.org/10.1063/1.4870642]
\end{abstract}

\section{INTRODUCTION}

It was shown some time ago that some complex non-Hermitian Hamiltonians may exhibit real eigenvalues. ${ }^{1,2}$ The conjecture that such intriguing feature may be due to unbroken paritytime (PT) symmetry ${ }^{3}$ gave rise to a very active field of research ${ }^{4}$ (and references therein). The first studied PT-symmetric models were mainly one-dimensional anharmonic oscillators ${ }^{3-6}$ and lately the focus shifted towards multidimensional problems. ${ }^{7-14}$ Among the most widely studied multidimensional PT-symmetric models we mention the complex versions of the Barbanis, ${ }^{7,8,10-14}$ and Hénon-Heiles ${ }^{7,12}$ Hamiltonians. Several methods have been applied to the calculation of their spectra: the diagonalization method, ${ }^{7-10,12,13}$ perturbation theory, ${ }^{7,9,10,12}$ classical and semiclassical approaches, ${ }^{7,8}$ among others. ${ }^{12,14}$ Typically, those models depend on a potential parameter $g$ so that the Hamiltonian is Hermitian when $g=0$ and non-Hermitian when $g \neq 0$. Bender and Weir ${ }^{13}$ conjectured that some of those models may exhibit phase transitions so that their spectra are real for sufficiently small values of $|g|$. Such phase transitions appear to be a high-energy phenomenon.

Multidimensional oscillators exhibit point-group symmetry (PGS). ${ }^{15,16}$ As far as we know such a property has not been taken into consideration in those earlier studies of the PT-symmetric models, except for the occasional parity in one of the variables. It is more than likely that PGS may be relevant to the study of the spectra of multidimensional PT-symmetric Hamiltonians. This paper is expected to be a useful contribution in that direction.

The research on non-Hermitian Hamiltonians has been mainly focussed on finding models with real spectrum. It is our purpose to show an example of PT-symmetric Hamiltonian with complex eigenvalues; a Hamiltonian with the phase transition at the Hermitian limit $g=0$. We will also show that PGS provides a simple and clear explanation of why the eigenvalues of such model are complex and not real as the other problems discussed so far.

In Sec. II we consider the dimensionless Schrödinger equation for a particle in a square box with the potential iaxy that is obviously PT-symmetric. In Sec. III we show that perturbation theory predicts that some of the eigenvalues are complex for sufficiently small values of $|a|$. In Sec. IV we analyze the eigenfunctions of the unperturbed and perturbed Hamiltonians from the point of

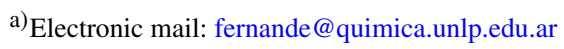


view of PGS and show why some eigenvalues are expected to be complex. In Sec. V we obtain the eigenvalues and eigenfunctions accurately by means of the diagonalization method and confirm the conclusions of Secs. III-IV. In Sec. VI we consider the particle in a square box with the potential $i a x y^{2}$ that resembles part of the potential of the PT-symmetric version of the Barbanis Hamiltonian. In this case PGS shows that PT symmetry may not be broken for sufficiently small values of $|a|$. This conclusion is confirmed by the diagonalization method. Finally, in Sec. VII we summarize the main results of the paper, draw conclusions, and put forward a somewhat general recipe for the appearance of complex eigenvalues in a given multidimensional non-Hermitian Hamiltonian.

\section{BOX MODEL WITH $\boldsymbol{C}_{2 v}$ POINT-GROUP SYMMETRY}

We first consider the Schrödinger equation $H \psi=E \psi$ with the dimensionless Hamiltonian operator,

$$
H=p_{x}^{2}+p_{y}^{2}+g x y
$$

and the boundary conditions

$$
\psi( \pm 1, y)=0, \psi(x, \pm 1)=0 .
$$

This Hamiltonian is Hermitian when $g$ is real and PT-symmetric when $g$ is imaginary. In fact, when $g=i a, a$ real, the Hamiltonian is invariant under two antiunitary transformations, ${ }^{17}$

$$
A_{x} H A_{x}=H, A_{y} H A_{y}=H
$$

generated by $A_{x}=P_{x} T$ and $A_{y}=P_{y} T$, where $T$ is the time-reversal operator ${ }^{18}$ and $P_{x}$ and $P_{y}$ are the parity transformations,

$$
\begin{aligned}
& P_{x}:\left(x, y, p_{x}, p_{y}\right) \rightarrow\left(-x, y,-p_{x}, p_{y}\right), \\
& P_{y}:\left(x, y, p_{x}, p_{y}\right) \rightarrow\left(x,-y, p_{x},-p_{y}\right) .
\end{aligned}
$$

It follows from Eq. (3) that

$$
H A_{x} \psi=A_{x} H \psi=A_{x} E \psi=E^{*} A_{x} \psi .
$$

That is to say, if $\psi$ is eigenfunction of $H$ with eigenvalue $E$ then $A_{x} \psi$ is eigenfunction with eigenvalue $E^{*}$. Obviously, the same conclusion applies to $A_{y} \psi$. When PT symmetry is unbroken,

$$
A_{x} \psi=\lambda \psi,|\lambda|=1,
$$

the corresponding eigenvalue is real. ${ }^{4}$ In a recent paper we have shown that the eigenvalue may be real even when this condition is manifestly violated. ${ }^{21}$ Later on we will discuss this point in more detail. All the Hamiltonians studied previously exhibit unbroken PT symmetry for sufficiently small values of $|g| .^{7-14}$ In what follows we show that the model depicted above behaves in a quite different way.

\section{PERTURBATION THEORY}

When $g=0$ the eigenvalues and eigenfunctions of the simple model described in Sec. II are those of the particle in a square box,

$$
\begin{aligned}
E_{m n}^{(0)} & =\frac{\left(m^{2}+n^{2}\right) \pi^{2}}{4}, m, n=1,2, \ldots, \\
\psi_{m n}^{(0)}(x, y) & =\varphi_{m n}(x, y)=\sin \left(\frac{m \pi(x+1)}{2}\right) \sin \left(\frac{n \pi(y+1)}{2}\right),
\end{aligned}
$$

and we appreciate that the eigenfunctions with $m \neq n$ are two-fold degenerate. There are accidental degeneracies that occur when $m_{1}^{2}+n_{1}^{2}=m_{2}^{2}+n_{2}^{2}$ but they are not relevant for the present discussion. 
For example, the three eigenfunctions $\psi_{71}^{(0)}, \psi_{17}^{(0)}$, and $\psi_{55}^{(0)}$ share the same eigenvalue but only the first two ones are consequence of the symmetry of the problem.

By means of perturbation theory it is quite easy to prove that some of the eigenvalues are complex for sufficiently small values of $|a|$. The perturbation correction of first order $E_{m n}^{(1)}$ vanishes when $n=m+2 j, j=0,1, \ldots$ but it is nonzero if $n=m+2 j+1$,

$$
\begin{aligned}
& E_{m n}^{(1)+}=\frac{256 m^{2}(2 j+m+1)^{2}}{\pi^{4}(2 j+1)^{4}(2 j+2 m+1)^{4}}, \\
& E_{m n}^{(1)-}=-\frac{256 m^{2}(2 j+m+1)^{2}}{\pi^{4}(2 j+1)^{4}(2 j+2 m+1)^{4}} .
\end{aligned}
$$

It is clear that for sufficiently small values of $|a|$ these levels behave approximately as linear functions of $g=i a$. In other words, the phase transition takes place at the Hermitian limit $a=0$. This result is different from that for the PT-symmetric oscillators studied so far that exhibit a vanishing perturbation correction of first order. ${ }^{7,12}$

\section{POINT-GROUP SYMMETRY}

We can understand the occurrence of complex eigenvalues more clearly from the point of view of PGS. Since the model is two-dimensional its behaviour with respect to the coordinate $z$ is irrelevant and, consequently, the choice of the point group is not unique. For the description of the unperturbed model $g=0$ we choose the point group $C_{4 v}$ with symmetry operations,

$$
\begin{aligned}
& E:(x, y) \rightarrow(x, y), \\
& C_{4}:(x, y) \rightarrow(y,-x), \\
& C_{4}^{3}:(x, y) \rightarrow(-y, x), \\
& C_{2}:(x, y) \rightarrow(-x,-y), \\
& \sigma_{v 1}:(x, y) \rightarrow(y, x), \\
& \sigma_{v 2}:(x, y) \rightarrow(-y,-x), \\
& \sigma_{d 1}:(x, y) \rightarrow(x,-y), \\
& \sigma_{d 2}:(x, y) \rightarrow(-x, y),
\end{aligned}
$$

where $C_{n}^{k}$ is a rotation by an angle $2 \pi k / n$ around an axis perpendicular to the center of the square box $\left(C_{4}^{2}=C_{2}\right)$ and $\sigma_{v}$ and $\sigma_{d}$ are vertical reflection planes. ${ }^{19,20}$ For simplicity we omit the transformation of the momenta when it is similar to that of the coordinates. The eigenfunctions form bases for the irreducible representations $\left\{A_{1}, A_{2}, B_{1}, B_{2}, E\right\}$ as indicated below:

$$
\begin{aligned}
A_{1} & :\left\{\varphi_{2 m-12 m-1}\right\},\left\{\varphi_{2 m-12 n-1}^{+}\right\}, \\
A_{2} & :\left\{\varphi_{2 m 2 n}^{-}\right\}, \\
B_{1} & :\left\{\varphi_{2 m 2 m}\right\},\left\{\varphi_{2 m 2 n}^{+}\right\}, \\
B_{2} & :\left\{\varphi_{2 m-12 n-1}^{-}\right\}, \\
E & :\left\{\varphi_{2 m-12 n}, \varphi_{2 n 2 m-1}\right\}, \\
\varphi_{m n}^{ \pm} & =\frac{1}{\sqrt{2}}\left(\varphi_{m n} \pm \varphi_{n m}\right), \\
m, n & =1,2, \ldots
\end{aligned}
$$


As expected some pairs of two-fold degenerate eigenfunctions form bases for the irreducible representation $E$. In addition to it, pairs of eigenfunctions with symmetry $A_{1}$ and $B_{2}\left(\varphi_{2 m-12 n-1}^{+}\right.$, $\left.\varphi_{2 m-12 n-1}^{-}\right)$as well as $A_{2}$ and $B_{1}\left(\varphi_{2 m 2 n}^{-}, \varphi_{2 m 2 n}^{+}\right)$are also degenerate.

When $g \neq 0$ a suitable point group is $C_{2 v}$ with symmetry operations $\left\{E, C_{2}, \sigma_{v 1}, \sigma_{v 2}\right\}$ and irreducible representations $\left\{A_{1}, A_{2}, B_{1}, B_{2}\right\}$. The eigenfunctions are linear combinations of the form,

$$
\begin{aligned}
\psi^{A_{1}} & =\sum_{m} \sum_{n}\left(a_{m n}^{A_{1}} \varphi_{2 m-12 m-1}+b_{m n}^{A_{1}} \varphi_{2 m 2 m}+c_{m n} \varphi_{2 m-12 n-1}^{+}+d_{m n} \varphi_{2 m 2 n}^{+}\right), \\
\psi^{A_{2}} & =\sum_{m} \sum_{n}\left(a_{m n}^{A_{2}} \varphi_{2 m-12 n-1}^{-}+b_{m n}^{A_{2}} \varphi_{2 m 2 n}^{-}\right), \\
\psi^{B_{1}} & =\sum_{m} \sum_{n} a_{m n}^{B_{1}} \varphi_{2 m-12 n}^{+}, \\
\psi^{B_{2}} & =\sum_{m} \sum_{n} a_{m n}^{B_{2}} \varphi_{2 m-12 n}^{-} .
\end{aligned}
$$

It is clear that the perturbation removes the degeneracy in such a way that the two-fold degenerate unperturbed eigenfunctions $E$ become the perturbed eigenfunctions of symmetry $B_{1}$ and $B_{2}$. As a result, every eigenvalue $E_{B_{1}}$ is the complex conjugate of an eigenvalue $E_{B_{2}}\left(E_{B_{2}}=E_{B_{1}}^{*}\right)$. As shown in Sec. III, the degeneracy of these levels is removed at first order of perturbation theory and it is not difficult to verify that the pair of integrals $\left\langle\varphi_{2 m 2 n-1}^{ \pm}|x y| \varphi_{2 m 2 n-1}^{ \pm}\right\rangle$give us exactly the perturbation corrections in Eq. (8). On the other hand, the degenerate unperturbed eigenfunctions of symmetry $A_{1}, A_{2}, B_{1}$, and $B_{2}$ become the perturbed eigenfunctions of symmetry $A_{1}$ and $A_{2}$. In this case the perturbation correction of first order vanishes and the degeneracy is removed at least at second order. If, as in the case of the models studied earlier by other authors, all the perturbation corrections of odd order vanish, ${ }^{7,12}$ then we may expect real eigenvalues for sufficiently small values of $|a|$.

PGS gives us a clear description of the occurrence of complex eigenvalues. If we take into account Eq. (5) and that $A_{x} \varphi_{2 m-12 n}^{+}=-\varphi_{2 m-12 n}^{-}$then we realize that $A_{x} \psi^{B_{1}}=\lambda_{B_{1} B_{2}} \psi^{B_{2}}$. We appreciate that PT symmetry is broken for all $|g| \neq 0$ and that $E_{B_{2}}=E_{B_{1}}^{*}$ as mentioned above. However, in principle it may be possible that both eigenvalues were real and degenerate as in the case of the rigid rotor studied in an earlier paper. ${ }^{21}$ In the present case we know that they are complex as shown in Sec. III. If we apply the same reasoning to the eigenfunctions of symmetry $A_{1}$ and $A_{2}$ we realize that PT-symmetry may not be broken for them because $A_{x} \psi^{A_{1}}=\lambda_{A_{1}} \psi^{A_{1}}$ and $A_{x} \psi^{A_{2}}=\lambda_{A_{2}} \psi^{A_{2}}$ (where $|\lambda|=1$ ) that follows from the fact that the symmetry-adapted basis functions are invariant or merely change sign under this antiunitary operation (and also under $A_{y}$ ).

\section{DIAGONALIZATION METHOD}

We can obtain sufficiently accurate eigenvalues and eigenfunctions of the box model by means of the diagonalization method. Diagonalization of the Hamiltonian matrix $\mathbf{H}$ in the basis set $\left\{\varphi_{m n}\right\}$ gives us the lowest eigenvalues of the Hamiltonian operator as well as the coefficients of the expansion of the eigenfunctions in the basis set

$$
\psi=\sum_{m} \sum_{n} a_{m n} \varphi_{m n}
$$

Alternatively, we can diagonalize Hamiltonian matrices $\mathbf{H}^{S}$ for each of the irreducible representations $S=A_{1}, A_{2}, B_{1}, B_{2}$ of the point group $C_{2 v}$ and thus obtain the corresponding sets of eigenfunctions (11) separately. In this case the dimension of the resulting secular equations is noticeably smaller.

It is well known that the coefficients of the characteristic polynomial generated by the full matrix $\mathbf{H}$ are real. ${ }^{22}$ The coefficients of the characteristic polynomials generated by $\mathbf{H}^{A_{1}}$ and $\mathbf{H}^{A_{2}}$ are polynomial functions of $g^{2}$ and therefore real. On the other hand, the coefficients of the characteristic polynomials generated by the matrices $\mathbf{H}^{B_{1}}$ and $\mathbf{H}^{B_{2}}$ are polynomial functions of $g$ and therefore complex. 

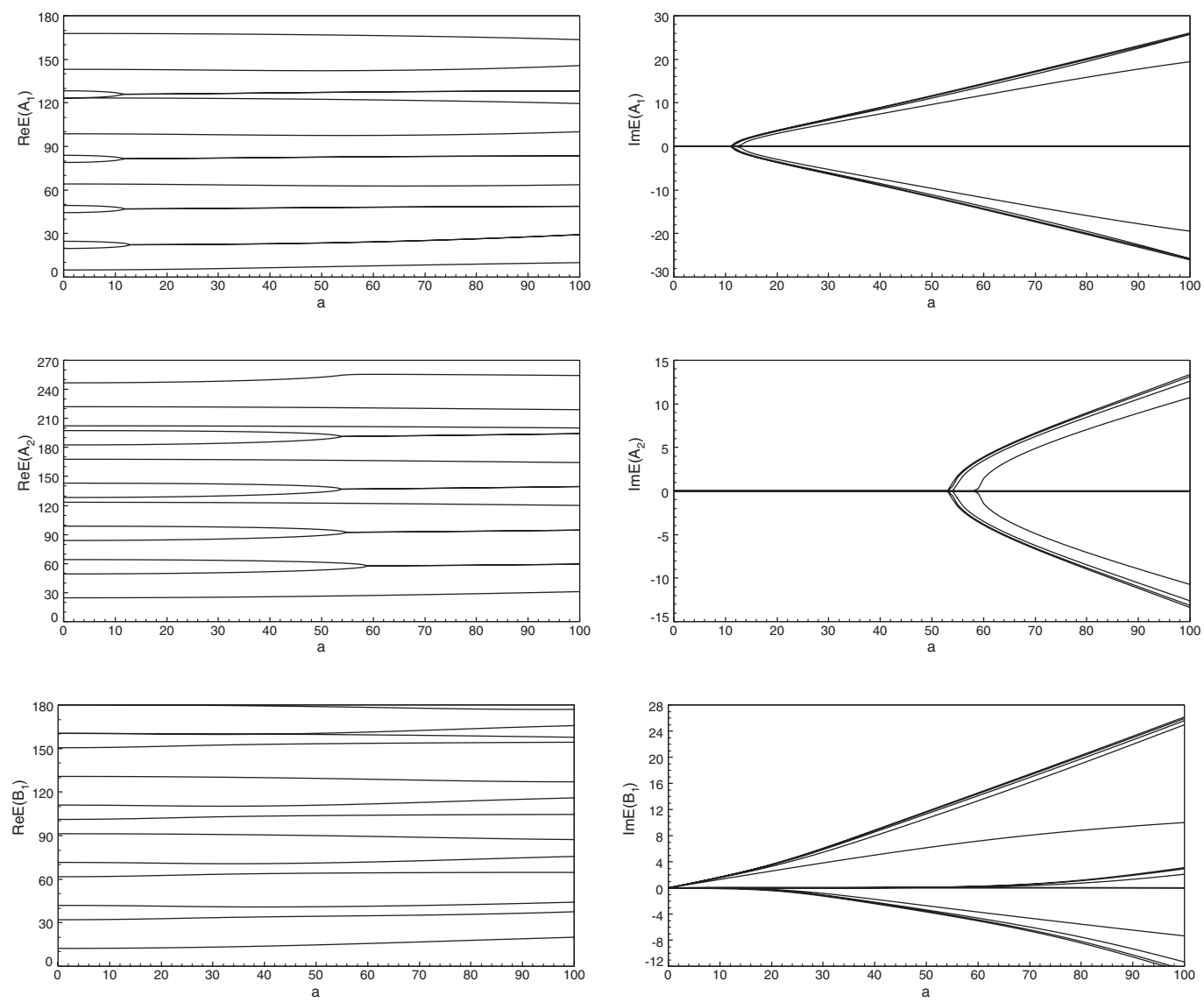

FIG. 1. First eigenvalues of the model (1).

Figure 1 shows the real and imaginary parts of the first eigenvalues of the Hamiltonian (1) for a wide range of values of $a$. The eigenvalues for symmetry $A_{1}$ and $A_{2}$ are real for sufficiently small values of $a$. Some pairs of them coalesce at critical values $a_{c}$ of the coupling constant and emerge as pairs of complex numbers for $a>a_{c}$. This occurrence of exceptional points is similar to that already found for other two-dimensional models. ${ }^{7-14}$ On the other hand, the eigenvalues for symmetry $B_{1}$ and $B_{2}$ are complex for all values of $a$. This kind of eigenvalues does not appear in those non-Hermitian Hamiltonians studied earlier. We say that the PT-symmetric Hamiltonian (1) exhibits a PT phase transition at the trivial Hermitian limit.

\section{BOX MODEL WITH $c_{2}$ POINT-GROUP SYMMETRY}

In order to illustrate the difference between present PT-symmetric model and those studied earlier, in this section we choose the particle in a square box with the interaction potential

$$
V(x, y)=g x y^{2},
$$

that resembles the one in the Barbanis Hamiltonian. ${ }^{7,8,10-14}$ In this case we may choose the point group $C_{2}$ with symmetry operations $\left\{E, C_{2}\right\}$, where $C_{2}:(x, y) \rightarrow(x,-y)$. The bases for the irreducible representations $\{A, B\}$ are $\left\{\varphi_{m 2 n-1}\right\}$ and $\left\{\varphi_{m 2 n}\right\}$, respectively. The antiunitary operator $A=P_{x} T$, where $P_{x}:\left(x, y, p_{x}, p_{y}\right) \rightarrow\left(-x, y,-p_{x}, p_{y}\right)$, leaves the Hamiltonian invariant when $g=$ $i a, a$ real. It follows from $A \varphi_{m n}=(-1)^{m+1} \varphi_{m n}$ and Eq. (5) that it is possible that $A \psi^{A}=\lambda_{A} \psi^{A}$ and $A \psi^{B}=\lambda_{B} \psi^{B}$; that is to say symmetry may be unbroken and the eigenvalues may be real for sufficiently small values of $|a|$. This observation is consistent with the fact that the perturbation 

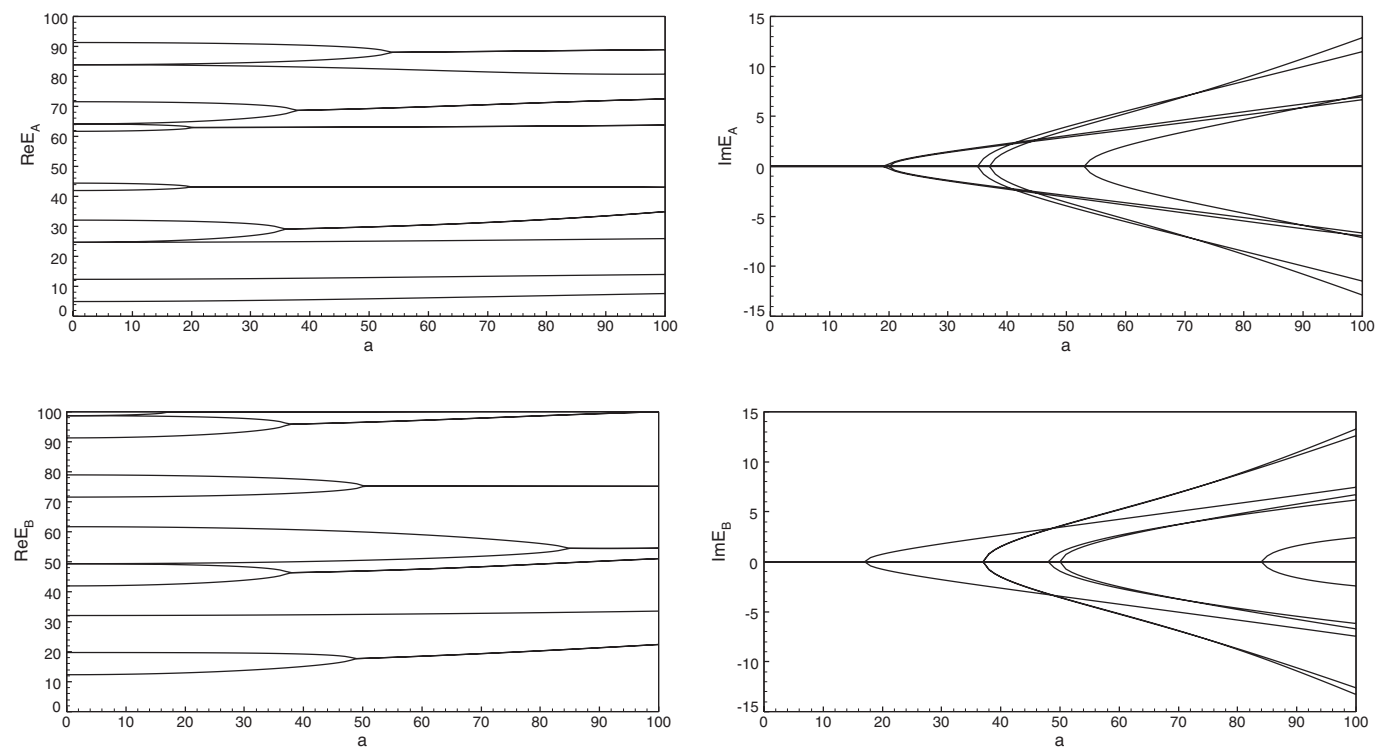

FIG. 2. First eigenvalues of the model (13).

correction of first order vanishes for all the states, which suggests that the perturbation expansion exhibits only even powers of $g$ as in the case of the Barbanis Hamiltonian. ${ }^{7,12}$

The results of the diagonalization method are shown in Figure 2. All the eigenvalues of both types of symmetry appear to be real for sufficiently small values of $|a|$ as expected from the argument based on PGS. Both sets of eigenvalues exhibit exceptional points where a pair coalesce and emerge as complex conjugate numbers. The main features of the spectrum of this model resemble those described in earlier problems.

\section{CONCLUSIONS}

In this paper we have discussed two non-Hermitian Hamiltonians with completely different spectra. Both are representative of a wider class of non-Hermitian Hamiltonians that depend on a parameter $g$ in such a way that they are Hermitian when $g=0$ and PT-symmetric for $g$ nonzero and imaginary (say, $g=i a, a$ real). The $C_{2}$ model is similar to those studied earlier that were chosen in such a way that all the eigenvalues are real when $0<a<a_{c} \cdot{ }^{7-14}$ It is said that they exhibit a PT phase transition at $a=a_{c}$ that was conjectured to be a high-energy phenomenon ${ }^{13}$ (at least for those examples). Consistent with real eigenvalues is the fact that their perturbation series exhibit only even powers of $g \cdot{ }^{7,9,10,12}$

The $C_{2 v}$ model is the main goal of this paper because it appears to exhibit complex eigenvalues for all values of $a \neq 0$. Therefore, in this case the PT phase transition takes place at the trivial Hermitian limit. The reason for this behaviour is that the degeneracy of the unperturbed Hamiltonian is broken at first order of perturbation theory so that the eigenvalues are almost linear functions of $g$ $=i a$ for sufficiently small values of $|a|$.

It has been our purpose to show that PGS is quite useful for the study of the spectra of such non-Hermitian Hamiltonians. The analysis of the eigenfunctions from such point of view clearly shows that PT symmetry is broken for those that are bases for some irreducible representations $\left(B_{1}\right.$ and $B_{2}$ in the present case). We may formulate the main ideas in a somewhat more general way. In general, the eigenfunctions are of the form

$$
\psi^{S}=\sum_{j} c_{j}^{S} \varphi_{j}^{S}
$$


where $S$ is an irreducible representation of the point group for the model. If $A \varphi_{j}^{S}=\lambda_{j}^{S} \varphi_{j}^{S}$, where $A$ is an antiunitary operation that leaves the Hamiltonian invariant, then it is possible that $A \psi^{S}=$ $\lambda_{S} \psi^{S}$ (unbroken PT symmetry) and the corresponding eigenvalues are real. All the models studied before exhibit this property. ${ }^{7-14}$ The eigenfunctions of symmetry $A_{1}$ and $A_{2}$ of present $C_{2 v}$ model also behave in this way. The situation is quite different in the case of the eigenfunctions of symmetry $B_{1}$ and $B_{2}$ that we may generalize it in the following way: when $A \varphi_{j}^{S}=\lambda_{k}^{S S^{\prime}} \varphi_{k}^{S^{\prime}}$ where $S^{\prime} \neq S$ then PT symmetry is broken $A \psi^{S}=\lambda_{S S^{\prime}} \psi^{S^{\prime}}$ and $E_{S}=E_{S^{\prime}}^{*}$. However, this relationship is not a rigorous proof that the eigenvalues are complex. In a recent paper we have shown that the eigenvalues of a PT symmetric rigid rotor are real even when PT-symmetry is broken as just indicated. ${ }^{21}$ In the case of present $C_{2 v}$ model the eigenvalues are in fact complex and for this reason we have decided to coin the term PT-symmetry broken by PGS.

Note added in the proof: S. Klaiman and L. S. Cederbaum also applied point-group symmetry to the study of some non-Hermitian oscillators in Phys. Rev. A 78, 062113 (2008). However, they only considered Abelian groups with one-dimensional irreducible representations and, consequently, all their models exhibited unbroken PT symmetry for sufficiently small $|\mathrm{g}|$.

\section{ACKNOWLEDGMENTS}

This report has been financially supported by PIP No. 11420110100062 (Consejo Nacional de Investigaciones Cientificas y Tecnicas, República Argentina).

${ }^{1}$ E. Caliceti, S. Graffi, and M. Maioli, Commun. Math. Phys. 75, 51 (1980).

${ }^{2}$ G. Alvarez, J. Phys. A 28, 4589 (1995).

${ }^{3}$ C. M. Bender and S. Boettcher, Phys. Rev. Lett. 80, 5243 (1998).

${ }^{4}$ C. M. Bender, Rep. Prog. Phys. 70, 947 (2007).

${ }^{5}$ F. M. Fernández, R. Guardiola, and M. Znojil, J. Phys. A 31, 10105 (1998).

${ }^{6}$ F. M. Fernández, R. Guardiola, J. Ros, and M. Znojil, J. Phys. A 32, 3105 (1999).

${ }^{7}$ C. M. Bender, G. V. Dunne, P. N. Meisinger, and M. Simsek, Phys. Lett. A 281, 311 (2001).

${ }^{8}$ A. Nanayakkara and C. Abayaratne, Phys. Lett. A 303, 243 (2002).

${ }^{9}$ A. Nanayakkara, Phys. Lett. A 304, 67 (2002).

${ }^{10}$ A. Nanayakkara, Phys. Lett. A 334, 144 (2005).

${ }^{11}$ H. Bíla, M. Tater, and M. Znojil, Phys. Lett. A 351, 452 (2006).

${ }^{12}$ Q.-H. Wang, Pramana J. Phys. 73, 315 (2009).

${ }^{13}$ C. M. Bender and D. J. Weir, J. Phys. A 45, 425303 (2012).

${ }^{14}$ C. R. Handy and D. Vrincenau, J. Phys. A 46, 135202 (2013).

${ }^{15}$ R. A. Pullen and A. R. Edmonds, J. Phys. A 14, L477 (1981).

${ }^{16}$ R. A. Pullen and A. R. Edmonds, J. Phys. A 14, L319 (1981).

${ }^{17}$ E. Wigner, J. Math. Phys. 1, 409 (1960).

${ }^{18}$ C. E. Porter, "Fluctuations of quantal spectra," in Statistical Theories of Spectra: Fluctuations, edited by C.E.Porter (Academic Press Inc., New York, 1965), p. 2.

${ }^{19}$ F. A. Cotton, Chemical Applications of Group Theory, 3rd ed. (John Wiley \& Sons, New York, 1990).

${ }^{20}$ M. Tinkham, Group Theory and Quantum Mechanics (McGraw-Hill Book Company, New York, 1964).

${ }^{21}$ F. M. Fernández and J. Garcia, "Critical parameters for non-hermitian Hamiltonians," e-print arXiv:1305.5164 [math-ph].

${ }^{22}$ F. M. Fernández, "On the real matrix representation of PT-symmetric operators,” e-print arXiv:1301.7639v3 [quant-ph]. 